\title{
Extended definitions of wake fields and their influence on beam dynamics
}

\author{
V. Danilov \\ ORNL, 104 Union Valley Road, Oak Ridge, Tennessee 37830
}

(Received 19 October 1999; published 31 January 2000)

\begin{abstract}
Based on experience gained from present machines, a new generation of accelerators with high intensities and low losses is being designed. For example, the design for the Spallation Neutron Source storage ring specifies fractional beam losses of the order of $10^{-4}$, so that even small instabilities or resonances can lead to the violation of this number. The purpose of this paper is to show that there exist potentially important beam fields, missing from standard analyses, that can lead either to instabilities or to large deviations of instability thresholds from their conventional values. Some of these fields and related effects, e.g., "fast damping," were discovered earlier and are presented here in more standard form as an extension of the wake field's definition. In addition, nonrelativistic collective phenomena are analyzed. It is shown that the nonrelativistic case could be significantly different from the relativistic case.
\end{abstract}

PACS numbers: 29.27.Fh, 41.85.Ew

\section{INTRODUCTION}

A complete representation of electromagnetic fields in vacuum chambers is very complicated, but for specific applications simplified descriptions often suffice. For example, to describe collective effects in practical cases one can often use simplified wake functions, which are electromagnetic forces integrated over the considered vacuum chamber element. In the case of transverse oscillations, one normally uses only the first term in the Taylor expansion of wakes over the transverse coordinate to calculate the linear eigenvalues of the collective motion. The integrated transverse force $F$ caused by a slight offset $r_{0}$ of the leading particle is conventionally related to the wake function $W$ by the following expression [1]:

$$
\int_{L} F d s=-q^{2} r_{0} W(z)
$$

where $q$ is the particle's charge and $z$ is the distance between head and tail particles.

It has been found [2] that consideration of fields proportional to the transverse velocity of the leading particle is necessary to explain one observed significant collective phenomenon, namely, "fast damping," which is an intensity dependent dipole oscillation damping that exists even for zero chromaticity. This term, proportional to the angle of the leading particle, has been included in some previous research [3]. It has been found recently [4] that in asymmetric structures the force from the tail particle depends on its own displacement, a fact that has proven to be significant [5]. One can summarize all the above statements in one general formula for the integrated transverse force in terms of the transverse offsets of the leading and trailing particles, $r_{0}$ and $r$, and their angles, $r_{0}^{\prime}$ and $r^{\prime}$ :

$$
\begin{aligned}
\int_{L} F d s= & -q^{2} r_{0} W(z)-q^{2} r_{0}^{\prime} G(z) \\
& -q^{2} r D(z)-q^{2} r^{\prime} A(z),
\end{aligned}
$$

where the functions $W(z)$ and $D(z)$ can be referred to as the driving and detuning wake functions, and the functions $G(z)$ and $A(z)$ as the angular ${ }^{1}$ driving and trailing wake functions.

In the next section, we present simplified calculations of the TEM line wake functions and give examples of driving and angular wakes for particles of arbitrary longitudinal velocity. The influence of all four terms on the beam dynamics is analyzed in the final section. It is shown also that nonrelativistic collective phenomena can be significantly different from those obtained in the ultrarelativistic case.

\section{SIMPLIFIED CALCULATION OF TEM LINE WAKES}

A TEM line is an element that displays both conventional and angular wakes. Moreover, one can easily calculate all the wakes for the nonrelativistic case. For this calculation we use the simplest model of two flat plates inserted into a vacuum chamber. We assume the plates are separated by a vertical distance $a$, their width is equal to $b \gg a$, and the total length is equal to $l$. The electric and magnetic fields $E$ and $H$ in the TEM line can be represented as

$$
\vec{E}=V(s, t) \vec{\varepsilon}, \quad \vec{H}=-I(s, t) \vec{h},
$$

where $\vec{\varepsilon}$ is proportional to the solution of the electrostatic problem of two plates with an opposite charge (in the line $\vec{\varepsilon}$ is the vertical vector with the total length $|\vec{\varepsilon}|=1 / a$ ) and $\vec{h}$ is the solution to the similar mangetostatic problem (in the line $\vec{h}$ is the horizontal vector with the length $|\vec{h}|=1 / b)$.

\footnotetext{
${ }^{1}$ The name is proposed by M. Blaskiewicz.
}

(c) 2000 The American Physical Society

014201-1 
The calculation presented below is simplified in the sense that we isolate the TEM fields and derive formulas only for them. The remaining fields are related to the space charge of the beam, and they give conventional contributions to the beam dynamics. ${ }^{2}$ To obtain the TEM field equations we substitute (3) into Maxwell equations and integrate over the TEM line cross section. This procedure corresponds to the standard expansion of the particle fields into the sum of the element's electromagnetic modes. The separated equations for $V, I$ are

$$
\begin{aligned}
& \frac{\partial V}{\partial s}+L_{0} \frac{\partial I}{\partial t}=0, \\
& \frac{\partial I}{\partial s}+C_{0} \frac{\partial V}{\partial t}=-\int_{\sigma_{\perp}} \vec{j} \cdot \vec{\varepsilon} d \sigma_{\perp},
\end{aligned}
$$

where $L_{0}=\mu_{0} a / b, C_{0}=\varepsilon_{0} b / a$ are the inductance and capacitance per length unit and $\vec{j}$ is the current density.

Consider a particle in an infinitely long TEM line with velocity $\nu_{s}$ parallel to the plates. Assume that at some moment of time (e.g., $t=0$ ) the particle receives a kick in the vertical direction, resulting in a small vertical velocity $\nu$. The vertical current density in this case is $j=$ $e \nu \delta(x) \delta(y) \delta\left(\nu_{s} t-s\right) \Theta(t)$, where $\Theta(t)$ is the Heaviside step function, and the solutions for $V, I$ are

$$
\begin{gathered}
V(s, t)= \begin{cases}-\frac{Z_{0}}{2(1+\beta) a} j_{0} \Theta(c t+s), & s<\nu_{s} t, \\
-\frac{Z_{0}}{2(1-\beta) a} j_{0} \Theta(c t-s), & s>\nu_{s} t,\end{cases} \\
I(s, t)= \begin{cases}+\frac{1}{2(1+\beta) a} j_{0} \Theta(c t+s), & s<\nu_{s} t, \\
-\frac{1}{2(1-\beta) a} j_{0} \Theta(c t-s), & s>\nu_{s} t,\end{cases}
\end{gathered}
$$

where $\beta=\nu_{s} / c, Z_{0}=\sqrt{\left(\mu_{0} / \varepsilon_{0}\right)}(a / b)$, and $j_{0}=e \nu$. This solution has several remarkable features.

First, it is proportional to the transverse velocity $\nu$. Second, it consists of two waves: a forward moving wave and a backward moving wave. In the ultrarelativistic limit the forward wave is very short, but its amplitude is large (proportional to $\gamma^{2}$ ). The backward moving wave is long and weak in the same limit. Note that the solution does not depend on the coordinates.

To find the solutions for the finite TEM line one uses the electric field at the line termination ports. The significant difference from the previous solution is that the TEM fields have large longitudinal components at the line ends, induced mostly by the longitudinal current. Besides, resistances $Z_{1}$ and $Z_{2}$ terminate both ends of the stripline. The equations (4) remain valid inside the stripline, but it is necessary to add the boundary conditions at the termination ports, namely,

$$
\begin{aligned}
& V=-\left.I Z_{1}\right|_{s=0} \quad \text { or } \quad \frac{-V}{Z_{1}}+C_{0} \int^{s} \frac{\partial V}{\partial t} d s=-\left.\int^{s} \int_{\sigma_{\perp}} \vec{j} \cdot \vec{\varepsilon} d \sigma_{\perp}\right|_{s=0}, \\
& V=\left.I Z_{2}\right|_{s=l} \quad \text { or } \quad \frac{V}{Z_{2}}+C_{0} \int^{s} \frac{\partial V}{\partial t} d s=-\left.\int^{2} \int_{\sigma_{\perp}} \vec{j} \cdot \vec{\varepsilon} d \sigma_{\perp}\right|_{s=l} .
\end{aligned}
$$

Assume for simplicity the matched line case, namely, $Z_{1}=Z_{2}=Z_{0}$. Assuming that the longitudinal coordinate of the particle is $s=0$ at time $t=0$, the solution for the fields inside the line $(0<s<1)$, proportional to the transverse velocity of the leading particle, is similar to (5):

$$
V(s, t)= \begin{cases}-\frac{Z_{0}}{2(1+\beta) a} j_{0}\left[\Theta(c t+s)-\Theta\left(c t+s-l \frac{1+\beta}{\beta}\right)\right], & s<\nu_{s} t, \\ -\frac{Z_{0}}{2(1-\beta) a} j_{0} \Theta(c t-s), & s>\nu_{s} t,\end{cases}
$$

where the solution again consists of two waves, absorbed without reflections at the terminating ports. Here we have omitted the solutions for the current $I$, which are easy to obtain from the solution for the voltage $V$.

\footnotetext{
${ }^{2} \mathrm{We}$ are mainly interested here in the angular transverse wake. It is related only to the fields, which absorb energy of transverse oscillations from the beam. For example, space charge fields in superconducting vacuum chambers have only conventional wake, even for an oscillating particle. That is why we consider only TEM fields here. rf cavities, which have modes with nonvanishing transverse electric field at the beam axis, have angular wakes similar to those in striplines.
}

The solution for the fields proportional to the coordinate displacement of the particle from the center of the line can be obtained as follows: First, substitute the solution for the voltage inside the TEM line $V^{\prime \prime}=f(c t-s)+$ $g(c t+s)$ into Eq. (6) to obtain

$$
\begin{aligned}
& \left.\frac{2 f(c t)}{Z 0}\right|_{s=0}=-\left.\int^{s} \int_{\sigma_{\perp}} \vec{j} \cdot \vec{\varepsilon} d \sigma_{\perp}\right|_{s=0}, \\
& \left.\frac{2 g(c t)}{Z 0}\right|_{s=l}=-\left.\int^{s} \int_{\sigma_{\perp}} \vec{j} \cdot \vec{\varepsilon} d \sigma_{\perp}\right|_{s=l} .
\end{aligned}
$$

The vertical electrostatic electric field $\vec{E}$ [see Eq. (3)] for the finite length TEM line can be written as $\vec{E}=$ $\vec{\varepsilon}(\Theta(s)-\Theta(s-l))$ with $\vec{\varepsilon}$ again equal to the vertical 
vector with the total length $1 / a$. For the longitudinal electric fields $E 1, E 2$ at both ends the Maxwell equations give

$$
E 1=\vec{\varepsilon}_{0} \cdot \vec{r} \delta(s), \quad E 2=-\vec{\varepsilon}_{0} \cdot \vec{r} \delta(s-l),
$$

with $\vec{r}$ equal to the particle transverse coordinate at the stripline terminations. Taking into account that the particle current density is $\vec{j}=e \delta\left(\nu_{s} t-s\right) \delta\left(y-y_{0}\right) \delta\left(x-x_{0}\right) \vec{\nu}$ one can combine (8) and (9) to obtain the solution for the $V^{\prime \prime}$ inside the stripline for $0<s<1$ :

$$
V^{\prime \prime}(s, t)=-\frac{e Z_{0} c}{2} \vec{\varepsilon}_{0} \cdot \vec{r}\left[\delta(c t-s)-\delta\left(c t+s-l \frac{1+\beta}{\beta}\right)\right] .
$$

The magnetic field can be obtained from the electric field. The function $I$ in Eq. (3) can be expressed in terms of $V$ in the following way:

$$
I(s, t)=-\int^{t} \frac{1}{c Z_{0}} \frac{\partial V}{\partial s} d \tau .
$$

The total electric field is the sum of $V^{\prime}$ and $V^{\prime \prime}$. To obtain the wake functions one can integrate the electromagnetic force on the particle trajectory over the stripline length:

$$
\begin{aligned}
& W(z)= \begin{cases}\frac{-\beta c Z_{0}}{2 a^{2}}[\Theta(-z)-\Theta(-z-(1+\beta) l)], & z<0, \\
\frac{\beta c Z_{0}}{2 a^{2}}[\Theta(z)-\Theta(z-(1-\beta) l)], & z>0,\end{cases} \\
& G(z)= \begin{cases}\frac{\beta c Z_{0}}{4 a^{2}} \Lambda((1+\beta) l+z), & z<0, \\
\frac{-\beta c Z_{0}}{4 a^{2}} \Lambda((1-\beta) l-z), & z>0,\end{cases}
\end{aligned}
$$

where function

$$
\Lambda(x)= \begin{cases}x, & x>0 \\ 0, & x<0\end{cases}
$$

The other wake functions, $D(z)$ and $A(z)$, are equal to zero in this case.

\section{THE INFLUENCE OF VARIOUS WAKES ON BEAM DYNAMICS}

In this section we present a brief description of the main impact on relativistic beam dynamics of the angular and detuning wakes. Assuming the bunch to consist of particles all having the same synchrotron amplitude, As, and a homogeneous distribution over the synchrotron phase (the so-called air-bag model), the transverse equation of motion reads

$$
\begin{aligned}
\frac{d^{2} x(\phi)}{d t^{2}}+\omega_{b}^{2} x(\phi) & =F_{x}(\phi), \\
F_{x}(\phi) & =-\frac{N q^{2}}{2 \pi \gamma m L} \int_{-|\phi|}^{|\phi|}\left[W(z) x\left(\phi^{\prime}\right)+D(z) x(\phi)+G(z) x^{\prime}\left(\phi^{\prime}\right)+A(z) x^{\prime}(\phi)\right] d \phi^{\prime}, \\
\frac{d}{d t} & =\frac{\partial}{\partial t}+\omega_{s} \frac{\partial}{\partial \phi}, \quad z=A s \cos \phi-A s \cos \phi^{\prime} .
\end{aligned}
$$

Here $\phi$ is the synchrotron phase, $\omega_{b}$ and $\omega_{s}$ are, respectively, the betatron and the synchrotron frequencies, and $N$ is the number of particles in the bunch. An expansion of the deviation $x(\phi)$ over the synchrotron harmonics

$$
x(\phi)=e^{-i \omega_{b} t} \sum_{n=-\infty}^{+\infty} x_{n} e^{-i \alpha \omega_{s} t+i n \phi}
$$

reduces (13) to a set of algebraic equations for eigenvectors with components $x_{n}$ and eigenvalues $\alpha$ :

$$
\begin{aligned}
x_{n}(\alpha-n)= & K \sum_{m=-\infty}^{+\infty} x_{m} K_{n m}, \quad K=\frac{N q^{2}}{2 \pi^{2} \gamma m \omega_{b} \omega_{s} L}, \\
K_{n m}= & \int_{0}^{\pi} \cos (n \phi) \int_{0}^{\phi} W(z) \cos \left(m \phi^{\prime}\right) d \phi^{\prime}+\int_{0}^{\pi} \cos [(n-m) \phi] \int_{0}^{\phi} D(z) d \phi^{\prime} \\
& -\int_{0}^{\pi} \cos (n \phi) \int_{0}^{\phi} \frac{i \omega_{b}}{\beta c} G(z) \cos \left(m \phi^{\prime}\right) d \phi^{\prime}-\int_{0}^{\pi} \cos [(n-m) \phi] \int_{0}^{\phi} \frac{i \omega_{b}}{\beta c} A(z) d \phi^{\prime},
\end{aligned}
$$


where the influence of the coherent interaction is taken to be small in comparison with the transverse focusing, $\alpha \omega_{s} \ll \omega_{b}$. We now consider all four contributions to the coefficient $K_{n m}$ from the TEM line fields.

\section{A. Driving wake contribution}

Because we analyze equations of motion with zero chromaticity, the integral of the driving wake is real. For small current it gives a coherent tune shift of all the modes. For large currents, different modes are coupled and the motion is unstable. For a short bunch in the relativistic case, the TEM line conventional wake is constant and is equal to $W(z)=-\beta c Z 0 / 2 a^{2}$. If one neglects the mode coupling, the coherent tune shift for the vertical motion for a mode with azimuthal number $n=0$ is

$$
\alpha=-K \frac{\pi^{2} \beta c Z_{0}}{4 a^{2}} .
$$

\section{B. Detuning wake contribution}

The flat TEM line detuning wake is equal to zero because the horizontal wake is equal to zero. In general, in flat geometry the detuning wake is opposite to the driving wake [5] and the coherent tune shift of the zero mode is equal to zero, $\alpha=0$. The effect of the detuning wake is stabilizing because it gives the betatron tune shift for the particles in the tail of the bunch. As a result, the transverse instabilities can be significantly suppressed [5].

\section{Driving angular wake contribution}

The contribution from the angular wakes is completely different from those of the driving and detuning wakes. In Eq. (15) the matrix elements from angular wakes are imaginary. Consider the driving angular wake, $G(z)=$ $\beta c Z_{0} l / 2 a^{2}$, for a relativistic short bunch in the stripline. This action of this wake is in the backward direction. The forward wake action is negligible in ultrarelativistic case, $1 \ll \gamma^{2} L_{b}$ (here and below $L_{b}$ stands for the bunch length). The tune shift for the zero mode is

$$
\alpha=-i K \frac{\pi^{2} Z_{0} l \omega_{b}}{4 a^{2}} .
$$

This result means that zero mode is damped in proportion to the number of particles in the bunch (so-called "fast damping"). This result, with the decrement proportional to the stripline length, can be found in Dikansky and Pestrikov [6] (see p. 251, Eq. 5.4.27). The small difference in formulas is related to the betatron phase advance at the stripline, which is neglected in our estimation.

\section{Trailing angular wake contribution}

The stripline trailing angular wake is zero. If we neglect the radiation force in the fields of the leading particle, this wake is equal to zero in all cases. However, the term proportional to the angle of trailing particle in Eq. (15) is related to the energy change due to the longitudinal wake, because the angle $x^{\prime}$ is equal to $p_{\perp} / p_{\|}$. In our model one can assume that the energy change associated with the collective fields is compensated by the rf system. Under this assumption the action of the last term in Eq. (15) averages into zero. In general, the trailing angular force can produce synchrobetatron resonances or damping similar to adiabatic damping.

\section{COLLECTIVE BEAM DYNAMICS IN THE NONRELATIVISTIC CASE}

Consider the nonrelativistic stripline driving wake $W(z)$ from Eq. (12) for small particle velocities, $\beta \ll 1$. It is exactly antisymmetric and the length of the wake in both directions is equal to the stripline length $l$. We now analyze three cases of beam dynamics using this wake. First is a simple two-particle model that already behaves quite differently from the relativistic case. Second, the equations for a bunch in a linac are solved, and, finally, the transverse mode coupling instability is analyzed for the bunch in a storage ring with synchrotron motion.

\section{A. Two-particle model}

The equations of motion for two particles in a stripline nonrelativistic wake, separated by less than the line length, are

$$
\begin{aligned}
\frac{d^{2} x_{1}}{d t^{2}}+\omega_{b}^{2} x_{1} & =F\left(x_{2}\right), \\
\frac{d^{2} x_{2}}{d t^{2}}+\omega_{b}^{2} x_{2} & =-F\left(x_{1}\right) \\
F(x) & =-\frac{q^{2}}{2 \pi m L} \frac{\beta c Z_{0}}{2 a^{2}} x=-C x .
\end{aligned}
$$

The squares of the eigenfrequencies $\omega$ are $\omega^{2}=$ $\omega_{b}^{2} \pm i C$. They are complex and the motion is unstable, contrary to the relativistic case where the trailing particle coordinate grows only linearly.

\section{B. Distributed bunch density in linac}

Now consider a bunch in a linac of length $L_{b}$, neglecting longitudinal motion and assuming constant linear density within the bunch. The stripline wake is antisymmetric with a step function on both sides of zero. For simplicity, we assume instead a sinusoidal antisymmetric wake with the wavelength equal to the bunch length, $W(z)=C \sin \left(2 \pi z / L_{b}\right)$ with $C \approx \beta c Z_{0} / 2 a^{2}$. The equation of motion is

$$
\begin{gathered}
\frac{d^{2} x(s)}{d t^{2}}+\omega_{b}^{2} x(s)=F_{x}(s) \\
F_{x}(s)=\frac{N q^{2} C}{2 \pi m L} \int_{0}^{L_{b}} \sin \left(2 \pi\left(s-s^{\prime}\right) / L_{b}\right) x\left(s^{\prime}\right) d s^{\prime}
\end{gathered}
$$


Here $L$ is the total linac length structure, treated as one stripline. The coordinate $x(s)$ can be expanded over spatial harmonics:

$$
\begin{aligned}
x(s)=\sum_{n=-\infty}^{+\infty} & \left(x_{n}(t) \cos \left(2 \pi n s / L_{b}\right)\right. \\
& \left.+y_{n}(t) \sin \left(2 \pi n s / L_{b}\right)\right) .
\end{aligned}
$$

Substitution of this formula into Eq. (19) yields

$$
\begin{aligned}
\frac{d^{2} x_{1}}{d t^{2}}+\omega_{b}^{2} x_{1} & =-k y_{1}, \\
\frac{d^{2} y_{1}}{d t^{2}}+\omega_{b}^{2} y_{1} & =k x_{1}, \\
k & =\frac{N q^{2} C}{4 \pi m L} .
\end{aligned}
$$

The other harmonics are uncoupled and oscillate at the betatron frequency. The frequencies of the first two harmonic oscillations are $\omega_{b}^{2} \pm i k$. Again, as in the case of the two-particle model, we have exponential growth. In the relativistic case a linear wake gives tail growth proportional to $\left(e^{t^{1 / 3}}\right)$ [1]. Asymptotically, this growth is much slower than that of the nonrelativistic case. This difference is related to the fact that tail particles interact with the head of the bunch and this coupling produces an exponential growth, which is related to the antisymmetric property of the wake function. For the symmetric wake functions the motion is stable-it provides only a coherent tune shift due to collective interaction.

\section{Mode coupling instability}

To analyze the difference between the instabilities for relativistic and nonrelativistic cases, we use Eq. (15), where, to get matrix elements $K_{m n}$, we integrate over forward and backward particles, since the wake is nonzero for positive arguments:

$$
K_{n m}=\int_{0}^{\pi} \cos (n \phi) \int_{0}^{\pi} W(z) \cos \left(m \phi^{\prime}\right) d \phi^{\prime} .
$$

Figure 1 shows two cases: a one-sided wake,

$$
W(z)= \begin{cases}C \sin (2 \pi z / a), & z<0 \\ 0, & z>0\end{cases}
$$

upper plot, and an antisymmetric two-sided wake,

$$
W(z)=C \sin (2 \pi z / a), \quad \forall z,
$$

lower plot, with $a$ equal to the amplitude of synchrotron oscillations for the "hollow beam" model. The horizontal scale is expressed in units of the parameter $Q=$ $N q^{2} C / 2 \gamma m \omega_{b} \omega_{s} L$. Both cases have almost the same threshold, but the imaginary parts of unstable modes grow much faster with intensity for the antisymmetric wake. Again, as for the linac instability, described by Eq. (21), the two-sided wake provides stronger head-tail coupling than the wake with backward action only; in the latter case this coupling comes only from the synchrotron motion.
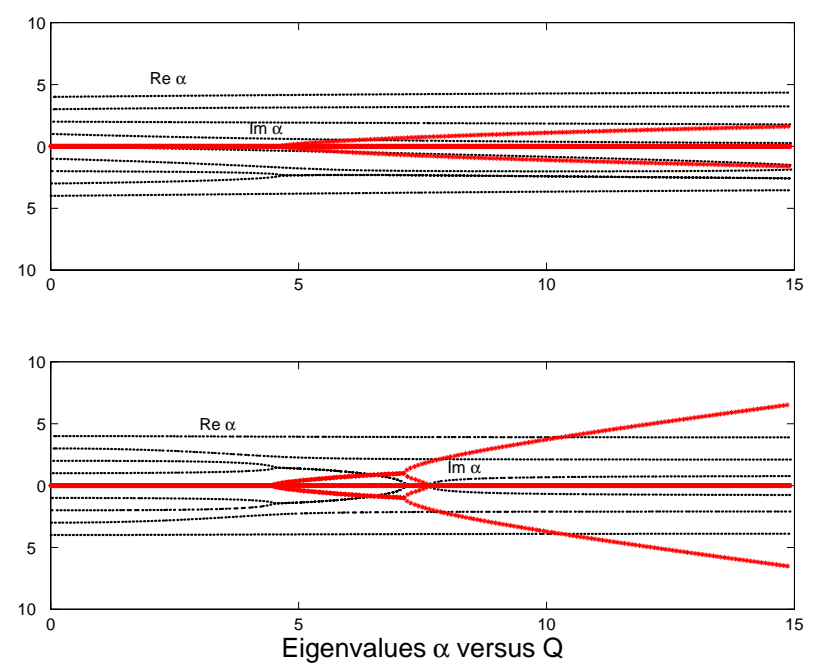

FIG. 1. (Color) Real (dotted lines) and imaginary (red lines) parts of the eigenfrequencies versus $Q$.

\section{CONCLUSION}

It can be concluded that, for some vacuum chamber elements, there exist beam-induced electromagnetic fields with special dependencies on particle coordinates and velocities. Those fields can significantly change the collective mode behavior. The collective phenomena can also be significantly different from extreme relativistic ones. These effects may be of importance for the new generation of high intensity facilities, such as the Spallation Neutron Source.

\section{ACKNOWLEDGMENTS}

The author acknowledges the support of the Division of Material Science, U.S. DOE, under Contract No. DEAC05-96OR22464 with LMER Corporation for ORNL, and is grateful to Mike Blaskiewicz and Sergey Kurennoy for interesting discussions. It also should be noted that most of the present material is derived from discussions several years ago with Novosibirsk accelerator physicists Eugene Perevedentsev and Alex Burov. Special thanks to Jeff Holmes for his numerous corrections of the manuscript.

[1] A. Chao, Physics of Collective Beam Instabilities (John Wiley \& Sons, New York, 1993).

[2] N. S. Dikansky et al., At. Energ. 22, 188 (1967).

[3] V.V. Danilov and E. A. Perevedentsev, in Proceedings of the 15th International Conference on High Energy Accelerators, 1992 (World Scientific, River Edge, NJ, 1992).

[4] S. Heifets, A. Wagner, and B. Zotter, SLAC Report No. SLAC/AP110, 1998.

[5] A. Burov and V. Danilov, Phys. Rev. Lett. 82, 2286 (1999).

[6] N. S. Dikansky and D. V. Pestrikov, The Physics of Intense Beams and Storage Rings (AIP, New York, 1994). 